\title{
miR-30a radiosensitizes non-small cell lung cancer by targeting ATF1 that is involved in the phosphorylation of ATM
}

\author{
YUYAN GUO, WENZE SUN, TUOTUO GONG, YANLAN CHAI, JUAN WANG, \\ BEINA HUI, YI LI, LIPING SONG ${ }^{*}$ and YING GAO* \\ Department of Radiation Oncology, The First Affiliated Hospital of Xi'an Jiaotong University, \\ Xi'an, Shaanxi 710061, P.R. China
}

Received September 2, 2016; Accepted January 24, 2017

DOI: $10.3892 /$ or.2017.5448

\begin{abstract}
Increasing number of studies report that microRNAs play important roles in radiosensitization. miR-30a has been proved to perform many functions in the development and treatment of cancer, and it is downregulated in non-small cell lung cancer (NSCLC) tissues and cells. This study was conducted to understand if miR-30a plays a role in the radiosensitivity of NSCLC cells. Radiosensitivity was examed by colony survival assay and tumor volume changing in vitro and in vivo, respectively. Bioinformatic analysis and luciferase reporter assays were used to distinguish the candidate target of miR-30a. qRT-PCR and western blotting were carried out to detect the relative expression of mRNAs and proteins. Cell cycle and cell apoptosis were determined by flow cytometry. Our results illustrated miR-30a could increase the radiosensitivity of NSCLC, especially in A549 cell line. In vivo experiment also showed the potential radiosensitizing possibility of miR-30a. Further exploration validated that miR-30a was directly targeting activating transcription factor 1 (ATF1). In studying the ataxia-telangiectasia mutated (ATM) associated effects on cell radiosensitivity, we found that miR-30a could reduce radiation induced $\mathrm{G} 2 / \mathrm{M}$ cell cycle arrest and may
\end{abstract}

Correspondence to: Professor Liping Song or Dr Ying Gao, Department of Radiation Oncology, The First Affiliated Hospital of Xi'an Jiaotong University, 277 Yanta West Road, Xi'an, Shaanxi 710061, P.R. China

E-mail: xaslp@126.com

E-mail: guoyuyan120@163.com; togaoying@163.com

${ }^{*}$ Contributed equally

Abbreviations: ATF1, activating transcription factor 1; ATM, ataxia-telangiectasia mutated; 3'UTR, 3' untranslated region; EMT, epithelial-mesenchymal transition; SDS-PAGE, sodium dodecyl sulfate-polyacrylamide gel electrophoresis; PVDF, polyvinylidene difluoride; IR, ionizing radiation; DSBs, DNA double-strand breaks

Key words: miR-30a, radiosensitivity, non-small cell lung cancer, activating transcription factor 1 , ataxia-telangiectasia mutated also affect radiation induced apoptosis. Together, our results demonstrated that miR-30a may modulate the radiosensitivity of NSCLC through reducing the function of ATF1 in phosphorylation of ATM and have potential therapeutic value.

\section{Introduction}

miR-30a has been implicated to function as tumor suppressor in various kinds of cancer (1), such as breast cancer (2), colon cancer (3), osteosarcoma (4), hepatocellular carcinoma (5), non-small cell lung cancer (NSCLC) (6), glioma (7), ovarian carcinoma (8) and renal clear cell carcinoma (9). Numerous studies have suggested that miR-30a could influence tumor progression through modulating cancer cell proliferation (10), migration, invasion (4), epithelial-mesenchymal transition (EMT) (2), apoptosis (5), autophagy (11) and other ways (12). A study on NSCLC tissues using miRNA microarray demonstrated that miR-30a was downregulated in both adenocarcinomas (14/20) and squamous cell carcinomas $(20 / 20)(\mathrm{P}=0.448)(13)$. Other studies (14) arrived to the same conclusion and revealed that its low expression was associated with cancer risk and indicated poor prognosis (15). In vitro experiment (16) also suggested miR-30a was downregulated in NSCLC A549 cells compared with BEAS-2B normal lung epithelial cells, overexpression of miR-30a could inhibit A549 lung cancer cell malignancy $(6,16)$. However, the exact role and underlying mechanism whereby miR-30a regulates the development and progression of NSCLC remains elusive.

MicroRNAs have been found to modulate tumor radiosensitivity in modulating a variety of pathways and molecules $(17,18)$. The primary ways that miRNAs modulate radiosensitivity were DNA damage repair, apoptosis, cell cycle checkpoint and tumor microenvironment (19). miR-124, miR-200c, miR-302 and miR-142 were found to affect the radiosensitivity of colorectal cancer (20), NSCLC (21), breast cancer (22) and malignant pediatric brain tumors (23), respectively. Moreover, a recent study firstly found that miR-30a could increase the radiosensitivity of prostate cancer cells (24). We did not find other studies concerning miR-30a and radiosensitivity. So, we investigated whether miR-30a could function as a radiosensitizer in NSCLC and its mechanism.

In this study, the effects of miR-30a on the radiosensitivity of NSCLC was studied in vitro and in vivo. Bioinformatic 
analysis and luciferase reporter assay illustrated that activating transcription factor 1 (ATF1) was a predicted target of miR-30a on its 3' untranslated region (3'UTR). Overexpression of miR-30a in NSCLC cell lines enhances the radiosensitivity of NSCLC, especially in A549 cells. Further investigation found ionizing radiation (IR)-induced G2/M cell cycle arrest was blocked by miR-30a and it may also affect the IR-induced apoptosis. These changes may be partly through binding to the 3'UTR of ATF1 mRNA, thereby affected the function of ATF1 in ataxia-telangiectasia mutated (ATM) phosphorylation process. Overall, our study indicated that miR-30a may be an important factor in influencing the radiosensitivity of NSCLC. More studies are needed to explore the precise molecular mechanism of miR-30a on regulating radiosensitivity in NSCLC.

\section{Materials and methods}

Cells and animal culture. A549 and H460 cells were obtained from the Center for Translational Medicine, Department of Xi'an Jiaotong University (Shaanxi, China). Cells were maintained in RPMI-1640 medium (Gibco Life Technologies, Carlsbad, CA, USA) containing 10\% fetal bovine serum (FBS; ExCell), cultured at $37^{\circ} \mathrm{C}$, humidified thermostat with $5 \% \mathrm{CO}_{2}$ atmosphere.

Five-week-old nude mice were used for tumor xenograft model and housed in the Center of Laboratory Animals of Xi'an Jiaotong University. The mice were all caged in specific pathogen free condition with constant temperature and humidity. Animals were randomly grouped to accept subcutaneous injection of A549 cells with lenti-GFP or lenti-miR-30a-5p or lenti-inhibitor stable expression. Animal experiments were authorized by the Institutional Animal Care and Use Committee of Xi'an Jiaotong University. Animal care abided by the rules of the Institutional Animal Care and Use Committee of Xi'an Jiaotong University.

MicroRNA transient transfection and lentiviral infection. miR-30a-5p agomir (50 nM), miR-30a-5p antagomir $(100 \mathrm{nM})$ and their negative control (50 or $100 \mathrm{nM})$ (GenePharma, Shanghai, China) (Table I) were transfected into cells, respectively, with Lipofectamine 3000 (Invitrogen Life Technologies, Carlsbad, CA, USA) transfection reagent following the manufacturer's instructions for 24 to $48 \mathrm{~h}$. Transfection efficiency were detected and cells were used in the assays descried below.

Lentiviral transduction vector pGMLV-MA2 or pGMLV-MI7 with miR-30a-5p overexpression or miR-30a-5p downregulation were co-transfected into HEK293T cells with packaging mix. Lentivirus particles were harvested $48 \mathrm{~h}$ after transfection. A549 cells (Genomeditech Co., Ltd., Shanghai, China) were infected using the recombinant lentivirus with $5 \mu \mathrm{g} / \mathrm{ml}$ polybrene.

RNA extraction and $q R T-P C R$ analysis. RNA extraction kit (Takara Bio, Inc., Shiga, China) was used to isolate total RNA and TRIzol (Invitrogen Life Technologies) to extract microRNA, following the manufacturer's instructions. PrimeScript RT Master Mix and Mir-X miRNA First-Strand Synthesis kit (both from Takara Bio, Inc.) were used to synthesise reverse
Table I. Sequences of hsa-miR-30a agomir, antagomir and their negative control.

\begin{tabular}{lc}
\hline Names & \multicolumn{1}{c}{ Sequences } \\
\hline hsa-miR-30a & 5'-UGU AAA CAU CCU CGA CUG GAA G-3' \\
agomir & 5'-UCC AGU CGA GGA UGU UUA CAU U-3' \\
Agomir NC & 5'-UUC UCC GAA CGU GUC ACG UTT-3' \\
& 5'-ACG UGA CAC GUU CGG AGA ATT-3' \\
hsa-miR-30a & 5'-CUU CCA GUC GAG GAU GUU UAC A-3' \\
antagomir & \\
Antagomir NC & 5'-CAG UAC UUU UGU GUA GUA CAA-3' \\
\hline
\end{tabular}

transcribed complementary DNA, respectively. SYBR Premix Ex Taq II and Mir-X miRNA qRT-PCR SYBR kit were used to perform qRT-PCR. U6 was the internal control. Primer sequences (5'-3') were as follows: hsa-miR-30a-5p, GTGTAAA CATCCTCGACTGGAAG; hsa-ATF1 forward, TTCTGGAG TTTCTGCTGCTGT and reverse, CCATCTGTGCCTGGAC TTG. All of the primers were synthesized by Sangon Biotech Co., Ltd. (Shanghai, China).

Dual-luciferase reporter assay. Fragments of ATF1 mRNA 3'UTR with either the sequence of miR-30a-5p binding site or its complementary bases were cloned in dual-luciferase report vector, obtaining pmirGLO-ATF1-wild and pmirGLO-ATF1-mutant recombinant plasmids (GenePharma). The two recombinant plasmids and pmirGLO-negative control were then transfected into A549 cells with miR-30a-5p agomir (50 nM). Thirty-six hours after transfection, Dual-Luciferase Reporter assay system (Promega, Madison, WI, USA) was used to measure the activity of luciferase.

IR. Linear accelerator (Siemens, Munich, Germany) was used for irradiation. Cells were treated with $200 \mathrm{cGy} / \mathrm{min}$ dose rate in room temperature to reach a required total dose using. IR group contain fifteen mice, consist of five randomly selected nude mice in each different miR-30a-5p expression group (10/group). Tumor-bearing mice in the IR group were treated with $2.0 \mathrm{~Gy}$ irradiation for 5 consecutive days from day 21 to 25 , and achieved a total dose of 10.0 Gy irradiation.

Colony formation assays. After 0, 2, 4, 6 and 8 Gy irradiation cells were incubation for 10 to 14 days. Formaldehyde of $4 \%$ was used to fix the cell clones and then stained with $1 \%$ crystal violet. Colonies of $\geq 50$ cells were counted and fitted to single target model using GraphPad Prism 5 (GraphPad Software, Inc., La Jolla, CA, USA).

Cell cycle and apoptosis analysis. For cell cycle assay, $70 \%$ ethanol was used to fix the harvested cells and placed at $-20^{\circ} \mathrm{C}$ overnight. Then incubated for $10 \mathrm{~min}$ in $50 \mu \mathrm{g} / \mathrm{ml}$ propidium iodide (PI) for analysis. Annexin V-PE/7-AAD apoptosis detection kit was used to test cell apoptosis, based on to the manufacturer's instructions. Cell cycle and apoptosis of the prepared cells were detected by flow cytometry (BD Biosciences, Franklin Lakes, NJ, USA). 
A

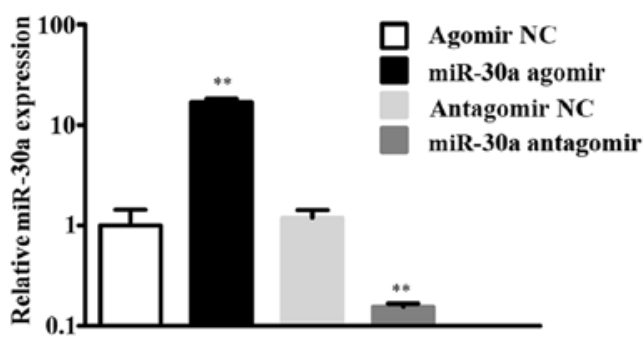

B

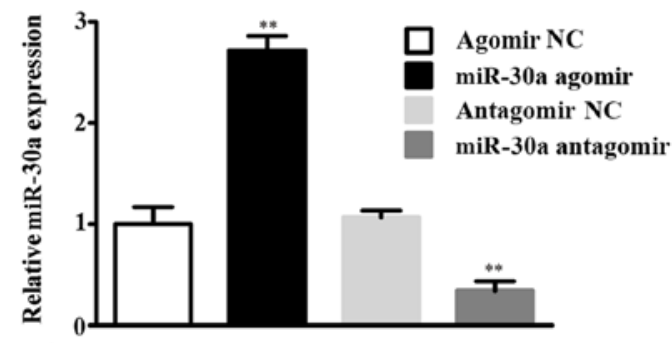

$\mathbf{E}$

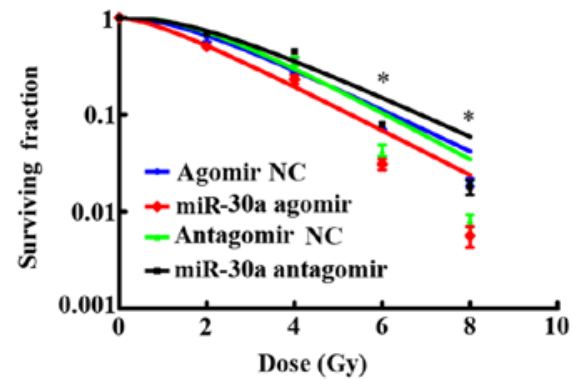

C

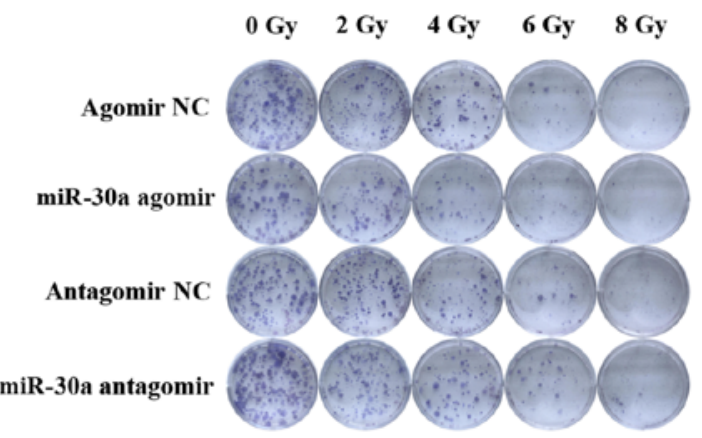

D

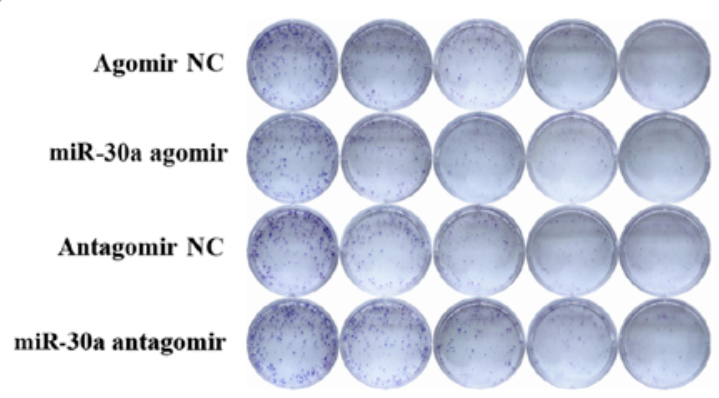

F

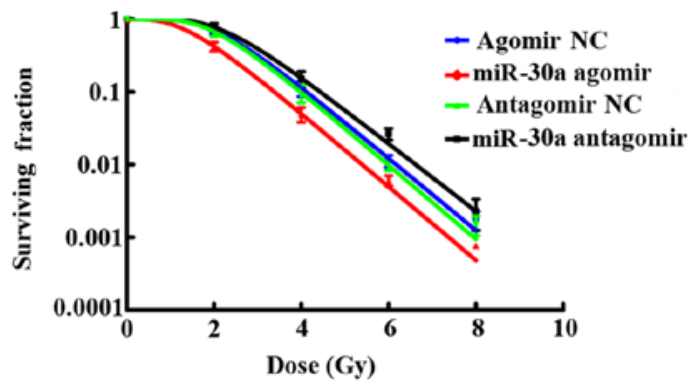

Figure 1. Relative miR-30a expression and colony survival analysis of A549 and H460 cells. (A and B) Relative miR-30a expression in (A) A549 and (B) H460 cells after transfected with hsa-miR-30a agomir, hsa-miR-30a antagomir and their negative controls, detected by qRT-PCR ("* P<0.01). (C and D) Representative images of colony survival assay after 0, 2, 4,6 and 8 Gy irradiation in (C) A549 and (D) H460 cells with different miR-30a expression. (E and F) Cell survival curves of (E) A549 and (F) H460 cells with different miR-30a expression after 0, 2, 4, 6 and 8 Gy irradiation, fitted with the single-hit multi-target model $(\mathrm{P}<0.05)$.

Western blotting. Cells were lysed in RIPA lysis buffer (BD Pharmingen, San Diego, CA, USA), protein concentration was measured by BCA reagent kit (Beyotime Institute of Biotechnology, Haimen, China). Sodium dodecyl sulfate-polyacrylamide gel electrophoresis (SDS-PAGE) was used to separate cellular lysates, then transferred to polyvinylidene difluoride (PVDF) membranes (Millipore, Boston, MA, USA). The transfected membranes were blocked in 5\% skim milk and incubated in $4^{\circ} \mathrm{C}$ overnight using the following primary antibodies: anti-ATF1 (1:1,000), anti-ATM (1:1,000), anti-ATM (phospho-1981; 1:5,000) (all from Abcam, Cambridge, MA, USA), anti-p53 (1:800), anti-p21 (1:500), anti-Bax (1:1,000) and anti-Bcl-2 (1:500) (all from Wanleibio, Shenyang, China), anti-GAPDH (1:3,000; Proteintech Group, Inc., Chicago, IL, USA). Then incubated with horseradish peroxidase-conjugated secondary antibody, goat anti-rabbit $(1: 5,000)$ or goat anti-mouse $(1: 5,000)$ (both from Cell Signaling Technology, Inc., Danvers, MA, USA). All bands were visualized with enhanced chemiluminescence (ECL) kit (Millipore).
Statistical analysis. Statistics of at least three different experiments were analyzed by GraphPad Prism 5. Results are presented as means \pm SEM. Statistical significance of two groups were tested by Student's t-test. P-value $<0.05$ was considered to have statistical significance.

\section{Results}

miR-30a enhances radiosensitivity of A549 and H460 cells. Colony survival assays were assessed to estimate the radiosensitivity of A549 and H460 cells. The two cell lines were treated with $0,2,4,6$ and 8 Gy radiation after transfected with miR-30a agomir $(50 \mathrm{nM})$ or miR-30a antagomir $(100 \mathrm{nM})$ or their negative controls (50 and $100 \mathrm{nM}$ ) for $36 \mathrm{~h}$, respectively. Besides, cell proliferation was evaluated using 3-(4,5-dimethylthiazol-2-yl)-2,5-diphenyltetrazolium bromide (MTT) assay after A549 cells were transfected with miR-30a agomir or miR-30a antagomir or their negative control. No statistical differences were found between the groups in 24,48 and 
A

Position 828-834 of ATF1 3'UTR 5'...UUUAAUAGAAUGGAAUGUUUACA. 1111111

B

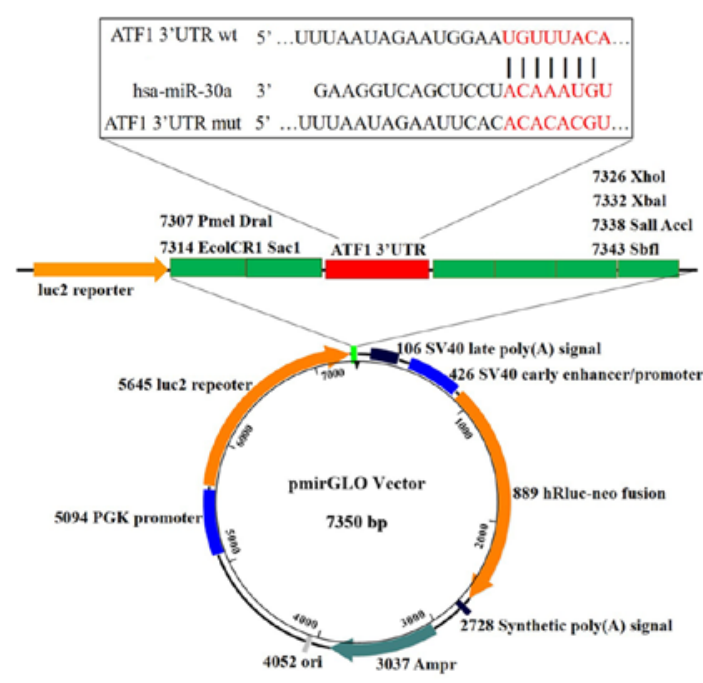

$\mathbf{E}$

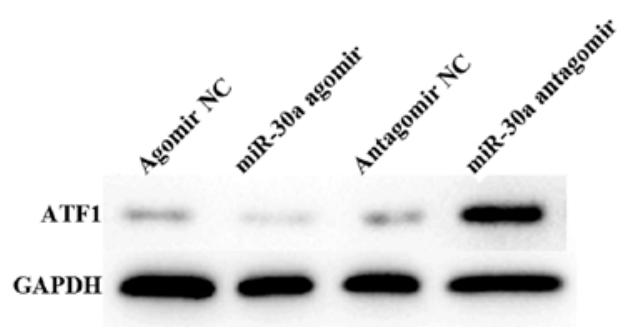

C

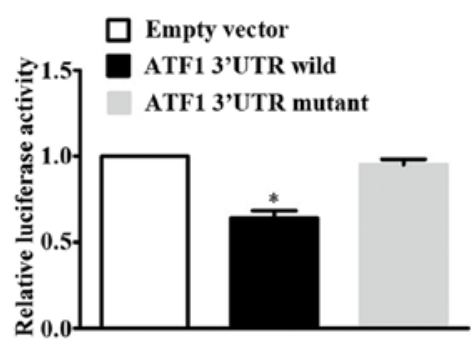

D

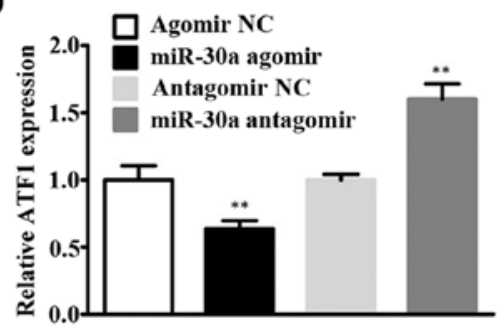

$\mathbf{F}$

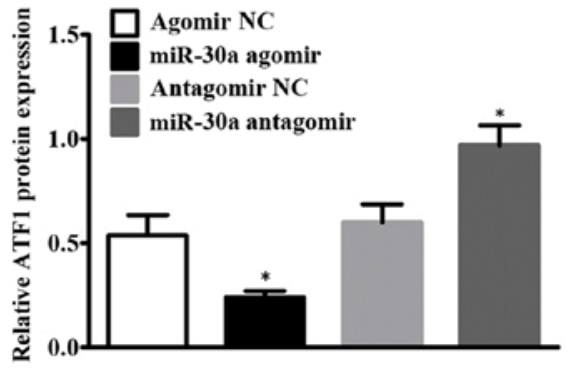

Figure 2. miR-30a directly targets the 3'UTR of ATF1. (A) The putative targeted sites of ATF1 in 3'UTR. (B) Schematic diagram of miR-30a targeting the 3'UTR of ATF1. (C) Relative luciferase activity in co-transfected groups of miR-30a agomir and pmirGLO vector, miR-30a agomir and pmirGLO ATF1-wild, miR-30a agomir and pmirGLO ATF1-mutant. (D) Relative ATF1 mRNA expression detected by qRT-PCR $\left({ }^{* *} \mathrm{P}<0.01\right)$. (E) Representative western blotting results. (F) Relative ATF1 protein expression in different miR-30a expression groups, relative ATF1 expression was downregulated in miR-30a agomir group $(0.24 \pm 0.05)$ compared with agomir NC group $(0.54 \pm 0.17)$, conversely, relative ATF1 expression was upregulated in miR-30a antagomir group (0.95 \pm 0.16$)$ compared with antagomir NC group $(0.60 \pm 0.15)\left({ }^{*} \mathrm{P}<0.05\right)$. 3'UTR, 3' untranslated region; ATF1, activating transcription factor 1.

$72 \mathrm{~h}$ (data not shown). miR-30a expression were examined by qRT-PCR and confirmed that the agomir and antagomir were transfected successfully $(\mathrm{P}<0.01)$ (Fig. 1A and B).

The miR-30a agomir groups of A549 cells showed a decrease of colony formation rate after radiation exposure compared to the controls, especially after $6 \mathrm{~Gy}(\mathrm{P}=0.0408)$ or $8 \mathrm{~Gy}(\mathrm{P}=0.0258)$ irradiation (Fig. $1 \mathrm{C}$ and $\mathrm{E})$. Conversely, the colony formation rate was increased in the miR-30a antagomir A549 cell groups than in the antagomir NC groups, 6 Gy $(\mathrm{P}=0.0103)$ and $8 \mathrm{~Gy}(\mathrm{P}=0.0451)$ also showed statistical significance (Fig. 1C and E). Results of the four groups in H460 cell line were in accordance with A549 cell line, but no statistical significance was found (Fig. 1D and F).

ATF1 expression is a target of miR-30a. In order to investigate the underlying mechanism of miR-30a affecting the radiosensitivity of NSCLC, we conducted bioinformatic analysis to predict the potential targets for miR-30a through searching PicTar, TargetScan and miRDB. We found that ATF1, which may also be associated with tumor radiosensitivity (25), was a predicted target of miR-30a (Fig. 2A).
Schematic diagram of miR-30a targeting the 3'UTR of ATF1 is shown in Fig. 2B.

Dual luciferase reporter assay was performed to further confirm that miR-30a directly target the 3'UTR of ATF1. The luciferase activity of pmirGLO-ATF1-wild was significantly decreased $(\mathrm{P}=0.0131)$, but pmirGLO-ATF1-mutant was not $(\mathrm{P}=0.2561)$, compared to pmirGLO-negative control group (Fig. 2C). Confirming that ATF1 could directly bind to the 3'UTR of miR-30a.

Furthermore, qRT-PCR and western blotting were assessed to examine if miR-30a could regulate the expression of ATF1 in A549 cell line. We found that ATF1 mRNA and protein were decreased in the miR-30a agomir group compared to the control group (Fig. 2D-F). Conversely, the ATF1 expression increased in the miR-30a antagomir group (Fig. 2D-F). These results further demonstrated that ATF1 was inversely regulated by miR-30a in the A549 cells.

miR-30a may enhance radiosensitivity of A549 cells through ATM pathway. Lentivirus systems were used to further explore the mechanism of miR-30a sensitizing radiation. A549 cells 
A

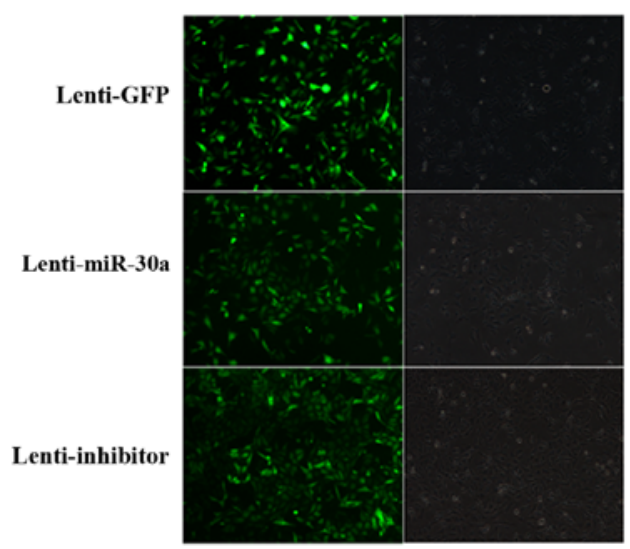

D

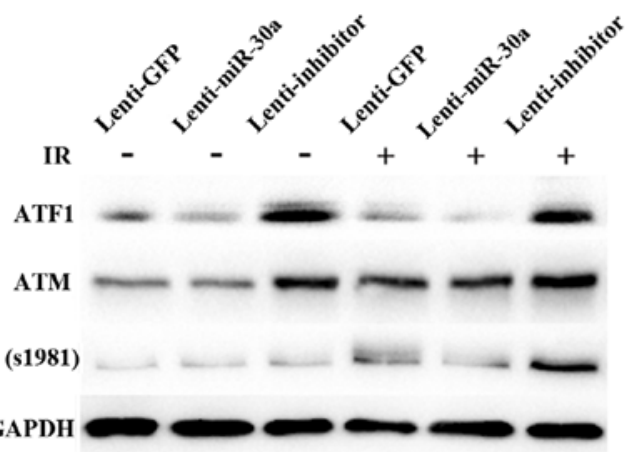

F

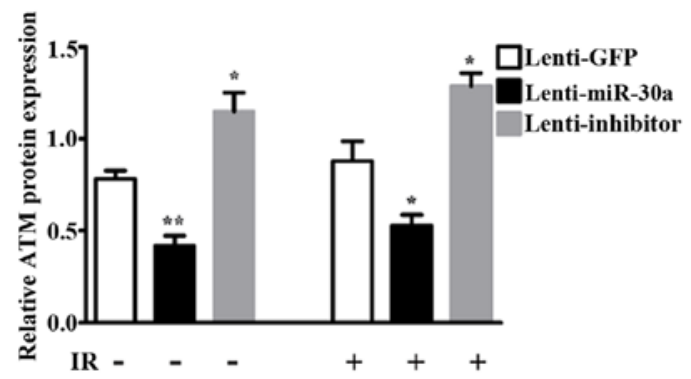

B

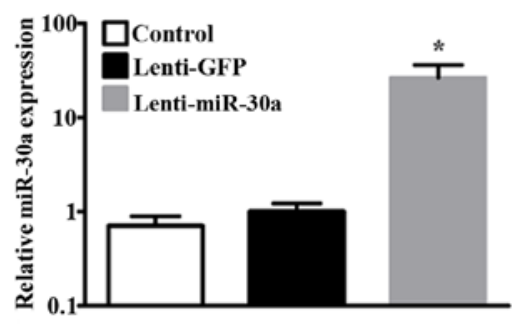

C

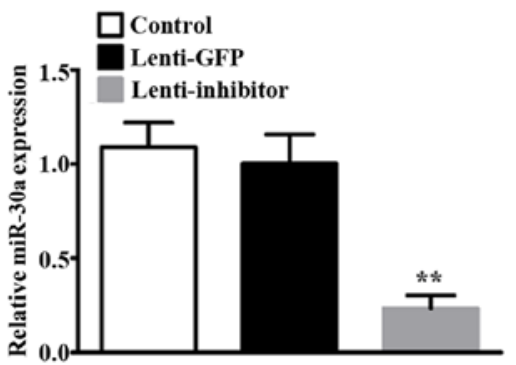

E

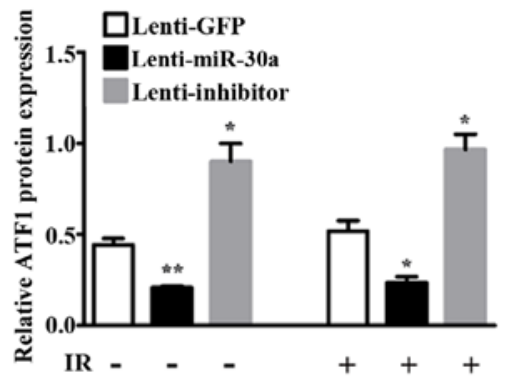

G

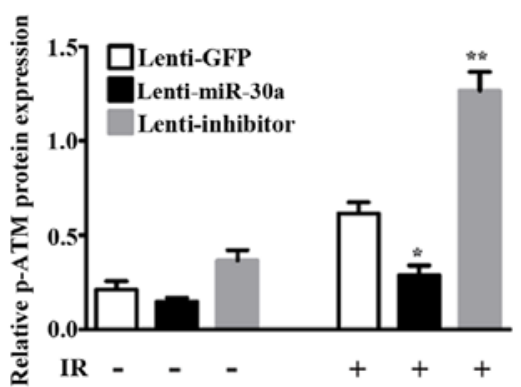

Figure 3. miR-30a affects the phosphorylation level of S1981 ATM after irradiation, consistent with ATF1. (A) Infection efficiency of lentiviruses estimated by the GFP tag and the corresponding bright field visual using a fluorescence microscope. (B and C) Relative miR-30a expression after lentivirus infection. (D) Representative western blotting results. (E) Relative ATF1 protein expression was downregulated in lenti-miR-30a A549 cells compared with lentiGFP A549 cells after 0 Gy $(0.21 \pm 0.01$ vs. $0.44 \pm 0.06)$ or 8 Gy $(0.24 \pm 0.05$ vs. $0.52 \pm 0.09)$ irradiation, lenti-inhibitor A549 cells showed the opposite results after 0 Gy $(0.90 \pm 0.17$ vs. $0.44 \pm 0.06)$ or $8 \mathrm{~Gy}(0.97 \pm 0.14$ vs. $0.52 \pm 0.09)$ irradiation. (F) Relative ATM protein expression was downregulated in lenti-miR-30a A549 cells compared with lenti-GFP A549 cells after 0 Gy $(0.42 \pm 0.09$ vs. $0.78 \pm 0.08)$ or 8 Gy $(0.53 \pm 0.10$ vs. $0.88 \pm 0.19)$ irradiation, lenti-inhibitor A549 cells showed the opposite results after 0 Gy $(1.15 \pm 0.17$ vs. $0.78 \pm 0.08)$ or 8 Gy $(1.29 \pm 0.12$ vs. $0.88 \pm 0.19)$ irradiation. (G) Phosphorylation of ATM at S1981 with 0 Gy irradiation were low and showed no statistical differences in lenti-miR-30a A549 cells $(0.15 \pm 0.04)$ or lenti-inhibitor A549 cells (0.37 \pm 0.10$)$ compared with lenti-GFP A549 cells (0.21 \pm 0.08$)$, after 8 Gy irradiation, IR-induced phosphorylation of ATM at S1981 was suppressed in lenti-miR-30a A549 cells (0.29 \pm 0.09$)$ but significantly induced in lenti-inhibitor A549 cells $(1.27 \pm 0.17)$, compared with lenti-GFP A549 cells $(0.61 \pm 0.10)\left({ }^{* *} \mathrm{P}<0.01\right.$; $\left.{ }^{*} \mathrm{P}<0.05\right)$. ATM, ataxia-telangiectasia mutated; ATF1, activating transcription factor 1.

with stable overexpression and downexpression of miR-30a were designated as lenti-miR-30a and lenti-inhibitor, respectively. A549 cells with stable expression of GFP was used as a control and named lenti-GFP. Infection efficiency at $48 \mathrm{~h}$ by fluorescence microscopy showed bright GFP tag in lentiviruses (Fig. 3A). Relative miR-30a expression by qRT-PCR showed miR-30a was significantly increased by lenti-miR30a $(\mathrm{P}=0.0108)$ (Fig. 3B) and decreased by lenti-inhibitor $(\mathrm{P}=0.0014)$ (Fig. 3C).
Western blotting results showed that ATF1 expression was downregulated in lenti-miR-30a cells and upregulated in lenti-inhibitor cells, compared with lenti-GFP cells (Fig. 3D and E). Given that ATM was an important and the first responder to DNA double-strand breaks (DSBs) (26) and by phosphorylation it involves in many IR-induced cell processes (27), we then detected ATM and p-ATM (S1981) expression. The results indicated that ATM protein expression and phosphorylation of ATM at S1981 corresponded 
A

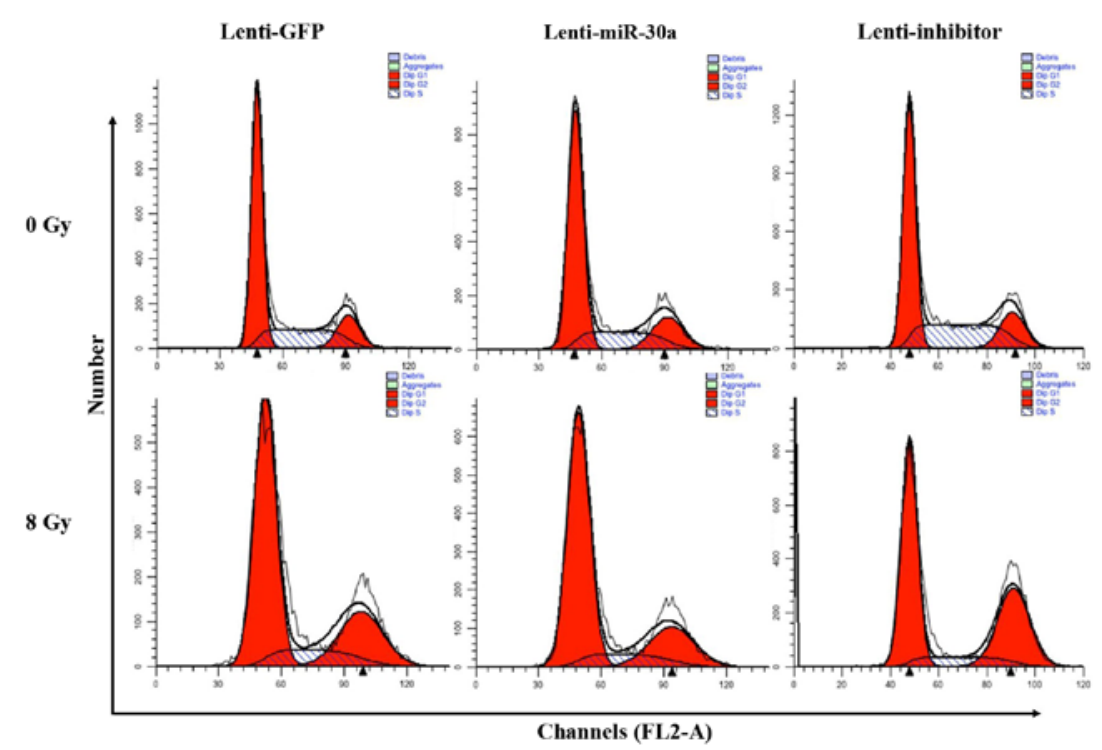

B

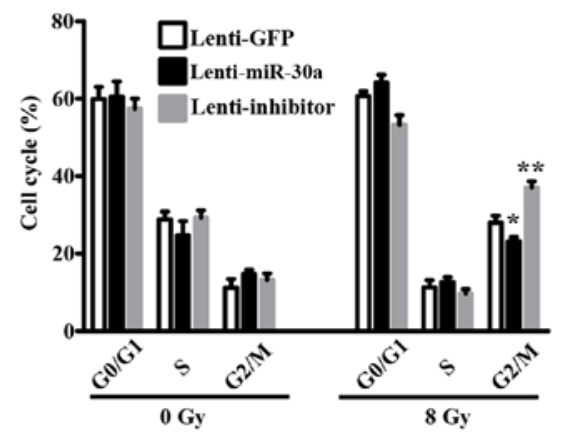

D

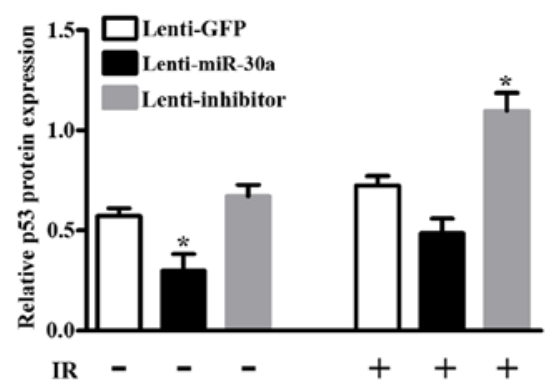

C

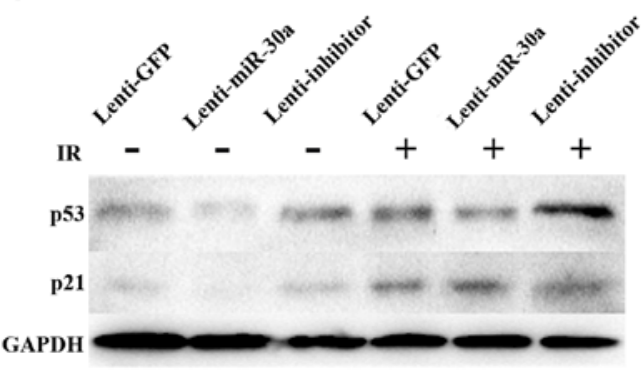

$\mathbf{E}$

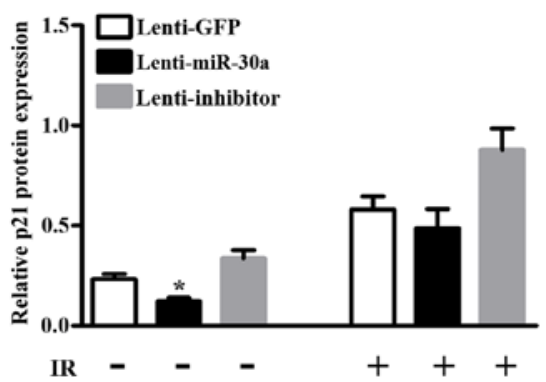

Figure 4. miR-30a blocks the radiation induced G2/M checkpoint arrest in A549 cell line. (A) Representative cell cycle distribution detected by flow cytometry. (B) Statistical image of cell cycle distribution. (C) Representative western blotting results of p53 and 21. (D) Relative p53 protein expression was downregulated in lenti-miR-30a A549 cells compared with lenti-GFP A549 cells after 0 Gy $(0.30 \pm 0.01$ vs. $0.57 \pm 0.07)$ or 8 Gy $(0.49 \pm 0.13$ vs. $0.73 \pm 0.08)$ irradiation, lentiinhibitor A549 cells showed the opposite results after 0 Gy $(0.67 \pm 0.10$ vs. $0.57 \pm 0.07)$ or 8 Gy $(1.10 \pm 0.16$ vs. $0.73 \pm 0.08)$ irradiation. (E) Relative p21 protein expression was downregulated in lenti-miR-30a A549 cells compared with lenti-GFP A549 cells after 0 Gy $(0.12 \pm 0.03$ vs. $0.23 \pm 0.04)$ or 8 Gy (0.48 \pm 0.17 vs. $0.58 \pm 0.11)$ irradiation, lenti-inhibitor A549 cells showed the opposite results after $0 \mathrm{~Gy}(0.31 \pm 0.07$ vs. $0.23 \pm 0.04)$ or 8 Gy $(0.88 \pm 0.18$ vs. $0.58 \pm 0.11)$ irradiation $\left({ }^{* *} \mathrm{P}<0.01 ;{ }^{*} \mathrm{P}<0.05\right)$.

with ATF1 (Fig. 3D, F and G). The expression of ATF1 and ATM showed no difference with 8 Gy irradiation or without irradiation. Phosphorylation of ATM at S1981 was very low without irradiation, and significantly increased after 8 Gy irradiation (Fig. 3G).

miR-30a enhances radiosensitivity by blocking the radiation induced G2/M checkpoint arrest in A549 cell line. To examine the impact of miR-30a on cell cycle progression and cell cycle distribution of A549 cells were measured by flow cytometry in lenti-miR-30a or lenti-inhibitor or lenti-GFP cells as contrast.
Cells after 0 or $8 \mathrm{~Gy}$ irradiation were collected to assess the cell cycle. Results revealed that in non-irritated group, cell cycle was not affected by miR-30a expression. However, after 8 Gy irradiation lenti-miR-30a decreased the proportion in $\mathrm{G} 2 / \mathrm{M}$ phase $(23.21 \pm 1.85 \%$ vs. $28.02 \pm 3.06 \%, \mathrm{P}=0.0251)$ and lenti-inhibitor contrarily showed increase in the proportion (37.05 $\pm 2.80 \%$ vs. $28.02 \pm 3.06 \%, \mathrm{P}=0.0075)$ (Fig. $4 \mathrm{~A}$ and $\mathrm{B}$ ).

In addition, western blotting results showed that miR-30a negatively influence IR-induced p53 expression, and the expression of p21 was suppressed (Fig. 4C). Taken together, the above results demonstrated that miR-30a may sensitize 
A
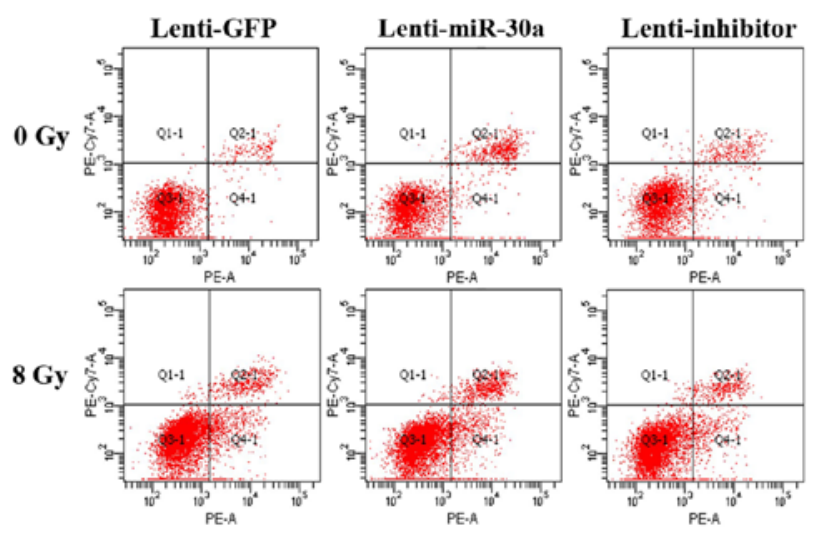

C

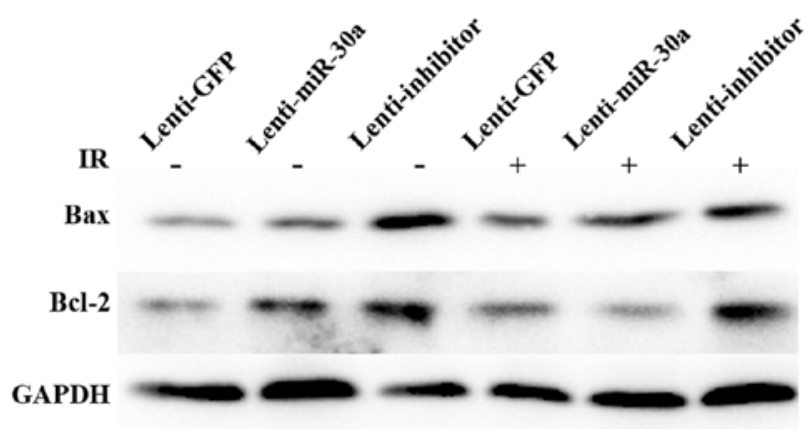

B

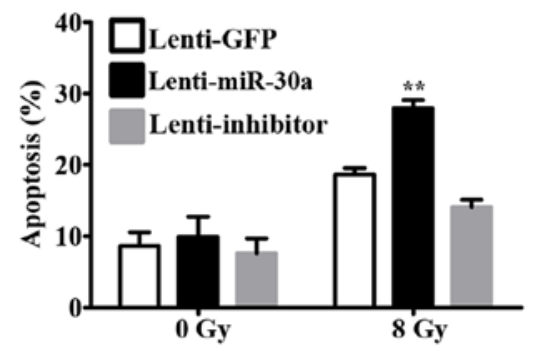

D

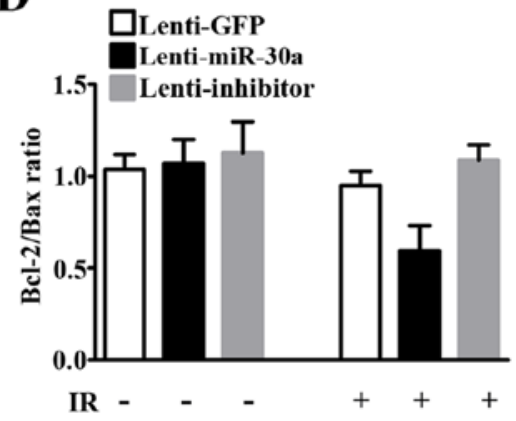

Figure 5. miR-30a may enhance irradiation-induced apoptosis of A549 cells. (A) Representative of cell apoptosis after 0 or 8 Gy IR detected by flow cytometry. (B) Statistical image of cell apoptosis $\left({ }^{* *} \mathrm{P}<0.01\right)$. (C) Representative western blotting results of Bax and Bcl-2. (D) Bcl-2/Bax ratio in lenti-miR-30a group (1.07 \pm 0.23$)$ or lenti-inhibitor group (1.13 \pm 0.29$)$ had no statistical differences compared with lenti-GFP group (1.04 \pm 0.14$)$ after 0 Gy irradiation. After $8 \mathrm{~Gy}$ irradiation, Bcl-2/Bax ratio was decreased in lenti-miR-30a group (0.59 \pm 0.23$)$ and increased in lenti-inhibitor group (1.09 \pm 0.14$)$ compared with lentiGFP group $(0.95 \pm 0.13)$, but not statistically significant. IR, ionizing radiation.

radiation by blocking the radiation induced $\mathrm{G} 2 / \mathrm{M}$ checkpoint arrest in A549 cells.

miR-30a may enhance irradiation-induced apoptosis of A549 cells. Furthermore, we detected the effects of miR-30a on apoptosis of A549 cells after irradiation by using flow cytometry. We found that miR-30a could not induce apoptosis without IR. The apoptosis rate was significantly increased after 8 Gy irradiation in all the three groups (Fig. 5A and B). Moreover, percentage of apoptosis in lenti-miR-30a cells was higher than the rate in lenti-GFP cells after 8 Gy irradiation $(27.93 \pm 2.00$ vs. $18.63 \pm 1.59 \%, \mathrm{P}=0.0026)$ (Fig. $5 \mathrm{~B})$. On the contrary, the apoptosis rate in lenti-inhibitor cells was modestly decreased, but not statistically significant $(14.1 \pm 1.73$ vs. $18.63 \pm 1.59 \%, \mathrm{P}=0.1409$ ) (Fig. 5B).

Since cell apoptosis after 8 Gy irradiation showed inconformity with p53 expression, we detected $\mathrm{Bcl}-2$ and Bax protein expression by western blotting. After IR, Bcl-2/Bax ratio in lentimiR-30a group was decreased compared with lenti-GFP group, but had no statistical significance and lenti-inhibitor group did not exhibit the desired result (Fig. 5C and D). Indicating that the apoptotic rate increase in the miR-30a upregulated group after IR may be partly involved in mitochondrial apoptotic pathway, but not in p53 apoptotic pathway.

These data illustrate that increasing radio-induced apoptosis may be another way in which miR-30a sensitizes the radiosensitivity of A549 cells. The precise regulatory mechanism is complicated and need further research.
miR-30a may enhance the sensitivity of A549 cells murine xenograft model to irradiation. To explore the radiosensitization potential of miR-30a in vivo, lenti-miR-30a and lenti-GFP cells were injected subcutaneously on the back of nude mice. Tumor growth was evaluated from the 7 th day after injection until the mice were sacrificed.

Our results illustrated that after IR tumor growth trend slowed down (Fig. 6A and B). Tumor volume in the lenti-miR-30a group were smaller than those derived from lenti-GFP group (Fig. 6C), and tumors in lenti-inhibitor group showed counter trends, but no statistical significance. Treatment dose and schedule may be the two main influencing factors. Moreover, when irradiating the tumor, the nude mice were completely exposed to X-ray. Nude mice in the IR group gradually showed a series of symptoms, the most obvious was weight loss, this may also influence the tumor size.

\section{Discussion}

MicroRNAs are important radiosensitivity regulators by interacting with the key molecules involved in radiosensitivity (19) and miR-30a has been found to act as a radiosensitizor in prostate cancer cells by targeting TP53INP1 and modulating autophagy (24). Thus, the potential therapeutic value of miR-30a attracted our interests in determine its function on NSCLC radiosensitization. Here, we found miR-30a functions as a sensitizer to irradiation in NSCLC cells, especially in A549 cells and may enhances the effect of radiation on tumors 
A

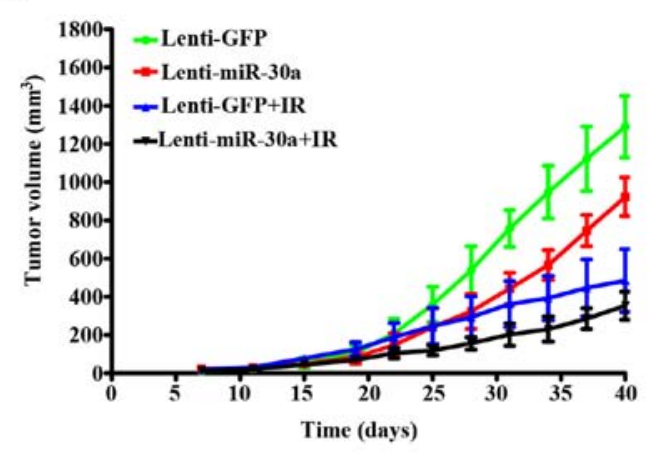

B

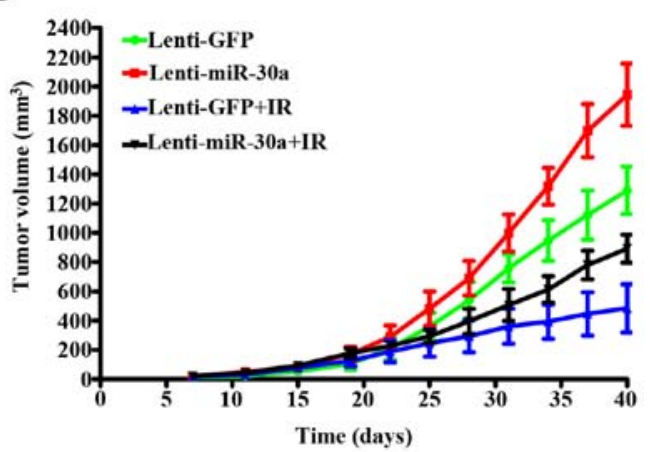

C

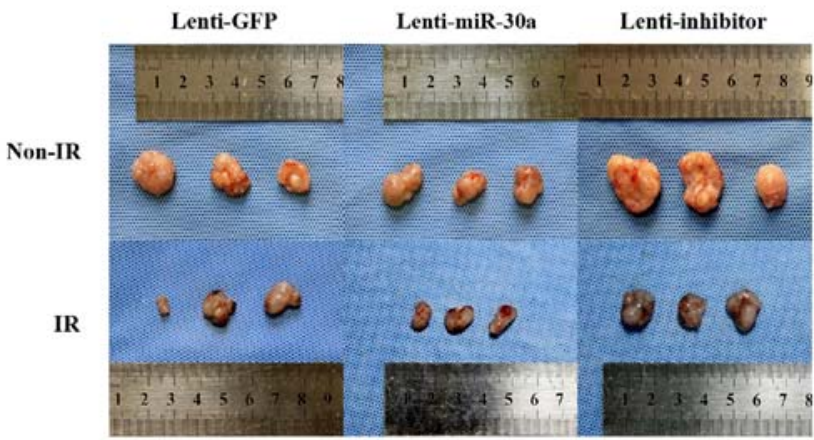

Figure 6. miR-30a may enhance the sensitivity of A549 cell murine xenograft model to irradiation. (A and B) Tumor volume growth curve in different miR-30a expression groups. (C) Representative tumors in different miR-30a expression and different treatment groups.

in nude mice. Furthermore, our data provide evidence for the potential role of miR-30a in suppressing the IR-induced $\mathrm{G} 2 / \mathrm{M}$ cell cycle arrest and increasing the IR-induced cell apoptosis.

The main target of IR is cellular DNA, ATM has a key role in the study of IR caused DNA damage (28). In response to DNA damage, by phosphorylation of ATM S1981, a series of downstream molecules can be actived to mediate cell cycle arrest, apoptosis (29) and initiate DNA repair (26). Shanware et al (25) announced that the downregulation of ATF1 could inhibit ATM expression synergistically. Interestingly, by using three public prediction databases we identified ATF1 as a potential target gene of miR-30a. The dual luciferase reporter assay, qRT-PCR and western blotting also proved that ATF1 is a direct target of miR-30a in the 3'UTR. Consistent with a previous study (25), we found that IR exposure neither affect the expression of ATM nor ATF1, but downregulation of ATF1 could reduce ATM expression and suppress IR induced ATM S1981 phosphorylation. These data suggested that by targeting ATF1, miR-30a could enhance the radiosensitivity of A549 cells through inhibiting the effect of ATF1 in IR induced ATM S1981 phosphorylation.

Since cell cycle arrest, DNA repair and apoptosis are the main ways that cancer cells react to IR through ATM (30), we further investigated the effect of miR-30a on these aspects after IR. Our results indicated that miR-30a could not alter cell cycle and apoptosis rate in non-irradiated A549 cells. While, miR-30a expression can increase IR-induced apoptosis and decrease IR-induced G2/M cell cycle arrest after 8 Gy IR. In response to IR induced DNA damage, phosphorylation of ATM can increase p53, either inducing DNA repair, cell cycle arrest (31), or apoptosis, thereby, maintain genomic stability (32) and this may also reduce the therapeutic effectiveness (33). p53 wild-type cell lines, when irradiating with ATM were downregulated, p53 cannot be retarded and lead to cell cycle checkpoint deficiency (1). In line with these documented studies, we noted in p53 wild-type A549 cells, p53 expression was consistent with the activation of ATM after IR. With p53 downregulation, cell cycle checkpoint was shortened, damaged cells cannot be eliminated in time, in this way, DNA repair ability can be decreased, thus radiosensitivity was enhanced. Moreover, with the accumulation of unrepaired, misrepaired and mutated DNA, the apoptosis can be subsequently increased, this may also partly cause the enhancing of radiosensitivity.

However, in human cancer, one individual miRNA could participate in the whole cancer procedure from initiation, progression to terminal by targeting hundreds of genes (34). They are involved in multiple pathways and could not only restrain but also accelerate cancer development (35). In our study, we surprisingly found that unlike A549, when combined with miR-30a, the colony survival of H460 showed a modest decrease, but no statistical difference with its control group. This may be associated with the modest miR-30a expression fold-change compared with A549 cells after miR-30a transfection (Fig. 1A and B). The in vivo study showed miR-30a can result in tumor volume regression, but still no statistical differences. Possibly this is due to the IR starting too late or the IR ceased too early or the IR dose was insufficient. The relationship between miR-30a expression and the time and dose of IR need further investigation to reveal the accurate role and profound underlying mechanism of miR-30a. 
In conclusion, our study indicated the importance of miR-30a in enhancing the radiosensitivity of A549 cell line by targeting ATF1, and association with the downregulation of ATM pathway, which may be a potential therapeutic factor of radiosensitization.

\section{Acknowledgements}

This study was supported by National Youth Science Fund Project (no. 81301937) from the National Natural Science Foundation of China.

\section{References}

1. Zhao JJ, Lin J, Zhu D, Wang X, Brooks D, Chen M, Chu ZB, Takada K, Ciccarelli B, Admin S, et al: miR-30-5p functions as a tumor suppressor and novel therapeutic tool by targeting the oncogenic Wnt/ $\beta$-catenin/BCL9 pathway. Cancer Res 74 : 1801-1813, 2014.

2. Chang CW, Yu JC, Hsieh YH, Yao CC, Chao JI, Chen PM, Hsieh HY, Hsiung CN, Chu HW, Shen CY, et al: MicroRNA-30a increases tight junction protein expression to suppress the epithelial-mesenchymal transition and metastasis by targeting Slug in breast cancer. Oncotarget 7: 16462-16478, 2016.

3. Baraniskin A, Birkenkamp-Demtroder K, Maghnouj A, Zöllner H, Munding J, Klein-Scory S, Reinacher-Schick A, Schwarte-Waldhoff I, Schmiegel W and Hahn SA: miR-30a-5p suppresses tumor growth in colon carcinoma by targeting DTL. Carcinogenesis 33: 732-739, 2012.

4. Zhang R, Yan S, Wang J, Deng F, Guo Y, Li Y, Fan M, Song Q, Liu H, Weng Y, et al: miR-30a regulates the proliferation, migration, and invasion of human osteosarcoma by targeting Runx2. Tumour Biol 37: 3479-3488, 2016.

5. Li WF, Dai H, Ou Q, Zuo GQ and Liu CA: Overexpression of microRNA-30a-5p inhibits liver cancer cell proliferation and induces apoptosis by targeting MTDH/PTEN/AKT pathway. Tumor Biol 37: 5885-5895, 2016.

6. Liu K, Guo L, Guo Y, Zhou B, Li T, Yang H, Yin R and Xi T: AEG-1 3'-untranslated region functions as a ceRNA in inducing epithelial-mesenchymal transition of human non-small cell lung cancer by regulating miR-30a activity. Eur J Cell Biol 94: 22-31, 2015.

7. Wang X, Wang K, Han L, Zhang A, Shi Z, Zhang K, Zhang H, Yang $\mathrm{S}, \mathrm{Pu} \mathrm{P}$, Shen $\mathrm{C}$, et al: PRDM1 is directly targeted by miR-30a-5p and modulates the $\mathrm{Wnt} / \beta$-catenin pathway in a Dkk1-dependent manner during glioma growth. Cancer Lett 331: 211-219, 2013.

8. Sestito R, Cianfrocca R, Rosanò L, Tocci P, Semprucci E, Di Castro V, Caprara V, Ferrandina G, Sacconi A, Blandino G, et al: miR-30a inhibits endothelin A receptor and chemoresistance in ovarian carcinoma. Oncotarget 7 : 4009-4023, 2016.

9. Huang QB, Ma X, Zhang X, Liu SW, Ai Q, Shi TP, Zhang Y, Gao Y, Fan Y, Ni D, et al: Down-regulated miR-30a in clear cell renal cell carcinoma correlated with tumor hematogenous metastasis by targeting angiogenesis-specific DLL4. PLoS One 8: e67294, 2013.

10. Zou Z, Ni M, Zhang J, Chen Y, Ma H, Qian S, Tang L, Tang J, Yao $\mathrm{H}$, Zhao C, et al: miR-30a can inhibit DNA replication by targeting RPA1 thus slowing cancer cell proliferation. Biochem J 473: 2131-2139, 2016.

11. Yu Y, Cao L, Yang L, Kang R, Lotze $M$ and Tang D: microRNA 30A promotes autophagy in response to cancer therapy. Autophagy 8: 853-855, 2012.

12. Ouzounova M, Vuong T, Ancey PB, Ferrand M, Durand G, Le-Calvez Kelm F, Croce C, Matar C, Herceg Z and Hernandez-Vargas H: MicroRNA miR-30 family regulates non-attachment growth of breast cancer cells. BMC Genomics 14: $139,2013$.

13. Zhu J, Zeng Y, Xu C, Qin H, Lei Z, Shen D, Liu Z and Huang JA: Expression profile analysis of microRNAs and downregulated miR-486-5p and miR-30a-5p in non-small cell lung cancer. Oncol Rep 34: 1779-1786, 2015.

14. Xie K, Wang C, Qin N, Yang J, Zhu M, Dai J, Jin G, Shen H, $\mathrm{Ma} \mathrm{H}$ and $\mathrm{Hu} \mathrm{Z}$ : Genetic variants in regulatory regions of microRNAs are associated with lung cancer risk. Oncotarget 7 : 47966-47974, 2016.
15. Tang R, Liang L, Luo D, Feng Z, Huang Q, He R, Gan T, Yang L and Chen G: Downregulation of miR-30a is associated with poor prognosis in lung cancer. Med Sci Monit 21: 2514-2520, 2015.

16. Yuan Y, Zheng S, Li Q, Xiang X, Gao T, Ran P, Sun L, Huang Q, Xie F, Du J, et al: Overexpression of miR-30a in lung adenocarcinoma A549 cell line inhibits migration and invasion via targeting EYA2. Acta Biochim Biophys Sin (Shanghai) 48: 220-228, 2016

17. Metheetrairut $\mathrm{C}$ and Slack FJ: MicroRNAs in the ionizing radiation response and in radiotherapy. Curr Opin Genet Dev 23: 12-19, 2013

18. Zhao L, Lu X and Cao Y: MicroRNA and signal transduction pathways in tumor radiation response. Cell Signal 25: 1625-1634, 2013.

19. Zhao L, Bode AM, Cao Y and Dong Z: Regulatory mechanisms and clinical perspectives of miRNA in tumor radiosensitivity. Carcinogenesis 33: 2220-2227, 2012.

20. Zhang Y, Zheng L, Huang J, Gao F, Lin X, He L, Li D, Li Z, Ding Y and Chen L: miR-124 radiosensitizes human colorectal cancer cells by targeting PRRX1. PLoS One 9: e93917, 2014.

21. Shi L, Zhang S, Wu H, Zhang L, Dai X, Hu J, Xue J, Liu T, Liang Y and Wu G: miR-200c increases the radiosensitivity of non-small-cell lung cancer cell line A549 by targeting VEGF-VEGFR2 pathway. PLoS One 8: e78344, 2013.

22. Liang Z, Ahn J, Guo D, Votaw JR and Shim H: MicroRNA-302 replacement therapy sensitizes breast cancer cells to ionizing radiation. Pharm Res 30: 1008-1016, 2013.

23. Lee YY, Yang YP, Huang MC, Wang ML, Yen SH, Huang PI, Chen YW, Chiou SH, Lan YT, Ma HI, et al: MicroRNA142-3p promotes tumor-initiating and radioresistant properties in malignant pediatric brain tumors. Cell Transplant 23: 669-690, 2014.

24. Xu CG, Yang MF, Fan JX and Wang W: miR-30a and miR-205 are downregulated in hypoxia and modulate radiosensitivity of prostate cancer cells by inhibiting autophagy via TP53INP1. Eur Rev Med Pharmacol Sci 20: 1501-1508, 2016.

25. Shanware NP, Zhan L, Hutchinson JA, Kim SH, Williams LM and Tibbetts RS: Conserved and distinct modes of CREB/ATF transcription factor regulation by PP2A/B56gamma and genotoxic stress. PLoS One 5: e12173, 2010.

26. Khoronenkova SV and Dianov GL: ATM prevents DSB formation by coordinating SSB repair and cell cycle progression. Proc Natl Acad Sci USA 112: 3997-4002, 2015.

27. Chou WC, Hu LY, Hsiung CN and Shen CY: Initiation of the ATM-Chk2 DNA damage response through the base excision repair pathway. Carcinogenesis 36: 832-840, 2015.

28. Rondeau S, Vacher S, De Koning L, Briaux A, Schnitzler A, Chemlali W, Callens C, Lidereau R and Bièche I: ATM has a major role in the double-strand break repair pathway dysregulation in sporadic breast carcinomas and is an independent prognostic marker at both mRNA and protein levels. Br J Cancer 112: 1059-1066, 2015.

29. Neumann J, Yang Y, Köhler R, Giaisi M, Witzens-Harig M, Liu D, Krammer PH, Lin W and Li-Weber M: Mangrove dolabrane-type of diterpenes tagalsins suppresses tumor growth via ROS-mediated apoptosis and ATM/ATR-Chk1/Chk2-regulated cell cycle arrest. Int J Cancer 137: 2739-2748, 2015.

30. Jiang H, Reinhardt HC, Bartkova J, Tommiska J, Blomqvist C, Nevanlinna H, Bartek J, Yaffe MB and Hemann MT: The combined status of ATM and p53 link tumor development with therapeutic response. Genes Dev 23: 1895-1909, 2009.

31. Zeng YC, Xing R, Zeng J, Xue M, Chi F, Xin Y, Fan GL, Wang HM, Duan QY, Sun YN, et al: Sodium glycididazole enhances the radiosensitivity of laryngeal cancer cells through downregulation of ATM signaling pathway. Tumour Biol 37: 5869-5878, 2016.

32. Gudkov AV and Komarova EA: The role of p53 in determining sensitivity to radiotherapy. Nat Rev Cancer 3: 117-129, 2003.

33. Bruno T, De Nicola F, Iezzi S, Lecis D, D'Angelo C, Di Padova M, Corbi N, Dimiziani L, Zannini L, Jekimovs C, et al: Che-1 phosphorylation by ATM/ATR and Chk2 kinases activates p53 transcription and the G2/M checkpoint. Cancer Cell 10: 473-486, 2006.

34. Berindan-Neagoe I, Monroig PC, Pasculli B and Calin GA: MicroRNAome genome: a treasure for cancer diagnosis and therapy. CA Cancer J Clin 64: 311-336, 2014.

35. Lin S and Gregory RI: MicroRNA biogenesis pathways in cancer. Nat Rev Cancer 15: 321-333, 2015. 